\section{A aplicabilidade atual dos índices simplificados de Viegas nos levantamentos epidemiológicos da cárie dentária}

\author{
The current applicability of Viegas simplified \\ indices to dental caries epidemiological surveys
}

\author{
${ }^{1}$ Faculdade de Odontologia \\ de Piracicaba, Universidade \\ Estadual de Campinas, \\ Piracicaba, Brasil. \\ Correspondência \\ M. L. R. Sousa \\ Departamento de \\ Odontologia Social, \\ Faculdade de Odontologia \\ de Piracicaba, Universidade \\ Estadual de Campinas. \\ Av. Limeira 901, Piracicaba, SP \\ 13414-900, Brasil. \\ luzsousa@fop.unicamp.br
}

\begin{abstract}
The aim of this study was to verify whether two simplified Viegas indices, "Method One" and "Method Two", could be recommended for epidemiological surveys. The sample was obtained from secondary data for 29 cities, totaling 2,378 epidemiological tests in 12-year-old schoolchildren. Considering mean DMFT in each city, three prevalence groups were obtained (low, moderate, and high), calculating the estimated value of simplified indices in each group. Moderate correlation in the three prevalence groups and similarity among the mean DMFT was found in "Method One", which describes first molar caries experience ( $p<0.05)$. "Method Two", which in addition to first molar caries experience included upper central incisor caries experience, showed similarity in mean DMFT only in low caries prevalence. The results allow one to conclude that the "Method One" simplified index can be used with low, moderate, and high dental caries prevalence, showing the first molar as an important indicator of caries level in populations.
\end{abstract}

Oral Health; Dental Caries; DMF Index
Silvia Cypriano 1

Maria da Luz Rosário de Sousa ${ }^{1}$ Ronaldo Seichi Wada 1

\section{Introdução}

Conforme a Lei 8.080 de 19 de setembro de 1990 (http://portalweb01.saude.gov.br/saude/ visao.cfm?id_area=169, acessado em 02/Dez/ 2002), os serviços de saúde devem ter por base alguns princípios, entre os quais, a utilização da epidemiologia para o estabelecimento de prioridades, alocação de recursos e a orientação programática. Desta forma, os estudos epidemiológicos devem contribuir para reduzir os problemas de saúde da população, melhorando o conhecimento da distribuição das doenças, dos fatores que determinam essa distribuição e das possibilidades de êxito das intervenções com a finalidade de avaliar o impacto destas ações em relação à melhoria das condições de vida da população 1 .

Considerando que a cárie dentária ainda se constitui em um importante problema de saúde pública, estudos que contribuam com a verificação da aplicação de índices e indicadores de saúde bucal devem ser realizados. A utilização de indicadores e índices para estudos epidemiológicos tem sido definida, prioritariamente, pela adequação dos mesmos para caracterizar os problemas, garantindo fidedignidade de dados, facilidade de aplicação e permitindo comparabilidade de resultados 2 .

O índice CPO-D, descrito por Klein \& Palmer ${ }^{3}$, mede a experiência de cárie na dentição permanente, e vem sendo largamente utilizado nos 
levantamentos epidemiológicos de saúde bucal, sendo recomendado pela Organização Mundial da Saúde (OMS) 4 para estudos de prevalência de cárie dentária. Em um grupo de indivíduos este índice resulta da divisão do número de dentes permanentes cariados, perdidos (extraídos devido à cárie) e obturados, pelo número de indivíduos examinados.

Segundo Viegas 5, os índices simplificados foram propostos com o objetivo de reduzir o tempo destinado à coleta dos dados, aumentando a produtividade e eficiência das pesquisas epidemiológicas. Dentre esses índices simplificados destacam-se dois desenvolvidos por Viegas 5, o "Método Um" (MID1) que consiste na experiência de cárie do primeiro molar permanente e o "Método Dois" (MID2), que acrescenta ao primeiro molar a experiência de cárie dos incisivos centrais superiores. Apesar destes índices simplificados serem viáveis e confiáveis para se estimar o índice CPO-D, não há relatos na literatura atual de levantamentos epidemiológicos utilizando esta metodologia.

McDonald \& Sheiham 6, analisando a distribuição de cárie nas diferentes superfícies dentais em populações jovens de 13 a 15 anos, em diferentes níveis de cárie, demonstraram que quando os níveis de cárie são baixos a maioria das lesões ocorrem na região oclusal ou em sulcos e fissuras. Com o aumento da média de dentes atacados pela cárie, as superfícies proximais são cada vez mais afetadas 7 . Por outro lado, Vasconcelos et al. ${ }^{8}$, ao verificarem a distribuição da cárie considerando a experiência por dente em prevalência muito alta, observaram que mais de $90,0 \%$ dos primeiros molares tinham experiência de cárie, mas os incisivos superiores também mostravam uma importante experiência, variando de 33,0 a 43,9\%, assim como os pré-molares.

Sendo assim, devido à queda nos índices de cárie observada nas últimas décadas 9,10,11,12,13, $14,15,16,17$, questiona-se se ainda hoje esses índices simplificados seriam aplicáveis e se refletiriam as diferentes situações de prevalência de cárie, pois esses índices consideram dentes específicos, em especial o primeiro molar.

Assim, o objetivo deste estudo foi verificar se os índices simplificados preconizados por Viegas 5, MID1 e MID2, podem ser indicados para levantamentos epidemiológicos de cárie dentária na atualidade.

\section{Metodologia}

A amostra para este estudo foi proveniente de dados secundários de levantamentos epide- miológicos em saúde bucal de trinta municípios localizados na região de Campinas, Estado de São Paulo, Brasil, obtidos no período compreendido entre os anos de 1998 e 2001, compreendendo diversos grupos etários da população. Em todos estes estudos, utilizou-se a metodologia proposta pela OMS 4 para o diagnóstico da condição dental, tratando-se de uma amostra probabilística sistemática, sem reposição, tendo sido obtida uma taxa de resposta de $84,1 \%$.

Após a obtenção da aprovação do Comitê de Ética em Pesquisa da Faculdade de Odontologia de Piracicaba, da Universidade Estadual de Campinas (no 089/2002), aferiu-se minuciosamente a digitação dos dados epidemiológicos, excluindo-se as fichas com dados incompletos e campos inválidos. Selecionou-se ao final, os exames epidemiológicos de 2.378 escolares de 12 anos de idade, verificando-se o CPO-D em cada localidade.

Os municípios foram então divididos de acordo com a escala de severidade 18 , que sugere que o índice CPO-D aos 12 anos possa ser utilizado como um indicador do estado de saúde entre diferentes populações. Essa escala indica uma prevalência muito baixa quando o CPO-D varia de 0 a 1,1, quando esta variação é de 1,2 a 2,6 considera-se baixa prevalência, quando o intervalo do CPO-D é de 2,7 a 4,4 a prevalência é moderada, sendo alta quando este intervalo é de 4,5 a 6,5 e finalmente a prevalência é muito alta quando o CPO-D é igual ou maior que 6,6.

Assim, definiu-se os grupos de prevalência. Um município foi considerado como sendo de prevalência muito baixa $(n=125)$. Treze municípios foram classificados com sendo de baixa prevalência de cárie, totalizando 1.141 exames epidemiológicos. Foram considerados como de moderada prevalência de cárie 14 municípios ( $\mathrm{n}=1.018$ ) e finalmente, dois municípios foram classificados como de alta prevalência de cárie ( $\mathrm{n}=188$ ). Nenhum município apresentou CPO$\mathrm{D} \geq 6,6$ e assim não se obteve amostra para a prevalência muito alta.

Verificou-se a seguir se as amostras em cada grupo de prevalência era suficiente para as análises. Utilizou-se a fórmula que toma como base a variabilidade (desvio-padrão) da média CPO-D, admitindo-se um erro de $5 \%$ e $95 \%$ de confiança 19. Excluiu-se assim os escolares do município de prevalência muito baixa, pois a amostra não foi suficiente para as análises, segundo o critério adotado para o presente estudo. Desta forma, obteve-se três grupos de prevalência - baixa, moderada e alta - com amostras adequadas para as análises. 
Os índices simplificados selecionados foram propostos por Viegas 5 . O índice simplificado MID1 estima o CPO-D por meio dos valores médios de experiência de cárie do primeiro molar inferior direito (dente 46), utilizando uma equação de regressão apropriada que para a idade de 12 anos é:

$$
y=-2,72+9,72 x
$$

onde: $y=\mathrm{CPO}-\mathrm{D}$ estimado e $x=$ valor médio do dente 46 , obtido pela divisão do número de dentes atacados pela cárie pelo número de indivíduos examinados.

O índice simplificado MID2 estima o valor do índice CPO-D por meio dos valores médios da experiência de cárie do primeiro molar inferior direito e dos dois incisivos centrais superiores (dentes 46, 11 e 21), utilizando uma equação de regressão que para a idade de 12 anos é:

$$
y=-0,01+4,35 x_{1}+5,52 x_{2}
$$

onde: $y=$ CPO-D estimado, $x_{1}=$ valor médio do dente 46 , e $x_{2}=$ valor médio dos dentes $11 \mathrm{e}$ 21 atacados pela cárie, dividido pelo total de crianças examinadas.

Calculou-se assim, o índice CPO-D, MID1 e MID2, individualmente e em cada grupo de prevalência, utilizando-se para tal o programa Epi Info versão 5.01; foi utilizado para a montagem do banco de dados e a entrada das informações. Uma versão específica do Programa Epibuco (http://www.saude.sp.gov.br/html/fr_ sbucal.htm, acessado em Abr/2003), com adaptações complementares desenvolvidas pelos autores deste, foi utilizada para o processamento e análise dos dados.

A distribuição da experiência de cárie por dente foi realizada por meio de freqüências simples, através da proporção de dentes com experiência de cárie (dentes diagnosticados como cariados, restaurados com cárie, restaurados e extraídos devido à cárie) em relação aos dentes irrompidos, para cada grupo de prevalência. Posteriormente, verificou-se também a proporção em relação ao total de dentes com experiência de cárie por grupo de dentes.

Para a análise estatística adotou-se o nível de significância de $5 \%$. O teste de Wilcoxon 20 foi utilizado para verificar se havia diferenças entre as médias do índice CPO-D e os índices estimados, com nível de significância de 5\%. A adoção de testes não paramétricos justifica-se, pois o índice CPO-D não apresentou distribuição normal em nenhuma das situações estudadas.

Para verificar a dispersão dos dados, utilizou-se diagramas de dispersão e a correlação de Pearson, e a escala proposta por Rowntree 21. O teste qui-quadrado também foi utilizado, adotando-se o nível de significância de 5\%.

\section{Resultados}

Nos 29 municípios pesquisados, o CPO-D variou de 1,76 a 6,20 aos 12 anos de idade, resultando em três grupos de prevalência de cárie, totalizando 1.141 indivíduos em baixa, 1.049 em moderada e 188 indivíduos em alta prevalência.

As médias do índice CPO-D segundo a classificação adotada podem ser observadas na Tabela 1. Também é possível observar os valores estimados dos índices simplificados e as suas diferenças em relação ao índice CPO-D, segundo as prevalências de cárie.

Por meio dos diagramas de dispersão dos dados, verificou-se a correlação entre o CPO-D e os índices simplificados, conforme a prevalência de cárie. Observou-se uma correlação moderada entre MID1 e o índice CPO-D em todas as prevalências estudadas. A correlação entre o CPO-D e o MID2 foi forte nos grupos de baixa e moderada prevalências de cárie, mas foi moderada no grupo de alta prevalência. Os valores de "r" estão apresentados na Tabela 1.

Com a finalidade de verificar se havia diferenças entre o CPO-D e os índices simplificados (MID1 e MID2) aplicou-se o teste de Wilcoxon. Diferenças estatisticamente significantes foram observadas entre o CPO-D e o MID2, em alta e moderada prevalências de cárie.

Para melhor compreensão dos resultados verificou-se ainda a distribuição da cárie segundo o dente, e as Figuras 1, 2 e 3 mostram essa distribuição em relação ao total de dentes permanentes irrompidos. Em todas as prevalências de cárie, os primeiros molares são os dentes mais afetados, seguido pelos segundos molares. Os incisivos centrais apresentaram baixo percentual de experiência de cárie.

Considerando o grupo de dentes atacados pela cárie, em baixa prevalência os molares concentram $86,8 \%$ do total de dentes com experiência de cárie, passando para $80,0 \%$ em prevalência moderada e $73,6 \%$ em alta prevalência, denotando uma tendência da cárie se concentrar nos molares em baixa prevalência ( $\mathrm{p}<0,001)$. Os pré-molares apresentaram as seguintes proporções: 8,0\% em baixa prevalência, $13,2 \%$ em moderada e $17,2 \%$ em alta prevalência de cárie $(\mathrm{p}<0,001)$. Nos incisivos, estas porcentagens variaram de $5,1 \%$ em baixa a $8,9 \%$ em alta prevalência ( $p=0,0001)$. O grupo menos afetado foi o de caninos, variando de 0,1 (baixa prevalência) a $0,3 \%$ (alta prevalência) ( $p=0,474)$, não diferindo entre os grupos avaliados. 
Média do índice CPO-D e dos índices simplificados de Viegas, segundo a prevalência de cárie aos 12 anos de idade, em 29 municípios. Região de Campinas, São Paulo, Brasil, 1998 a 2001.

\begin{tabular}{lccc}
\hline Variáveis & Baixa & $\begin{array}{c}\text { Prevalência de cárie } \\
\text { Moderada }\end{array}$ & Alta \\
\hline CPO-D (dp) & $2,29(2,36)$ & $3,36(3,12)$ & $5,54(3,77)$ \\
MID1 (df) & $2,09(-0,20)$ & $3,18(-0,18)$ & $5,30(-0,24)$ \\
Correlação de Pearson $(r)$ & 0,68 & 0,63 & 0,49 \\
Valor de p & 0,169 & 0,998 & 0,882 \\
MID2 (df) & $2,48(0,19)$ & $3,25(-0,11)$ & $4,79(-0,75)$ \\
Correlação de Pearson $(r)$ & 0,72 & 0,72 & 0,57 \\
Valor de p & 0,332 & $0,006^{*}$ & $<0,0001^{*}$ \\
\hline
\end{tabular}

$\mathrm{dp}=$ desvio-padrão; $\mathrm{df}=$ diferença entre $\mathrm{o}$ índice CPO-D e seus respectivos índices simplificados de cárie dentária.

* diferença estatisticamente significante (teste de Wilcoxon).

Figura 1

Proporção de dentes com experiência de cárie dentária, em relação aos dentes irrompidos, em municípios com baixa prevalência de cárie aos 12 anos. Região de Campinas, São Paulo, Brasil, 1998-2001.

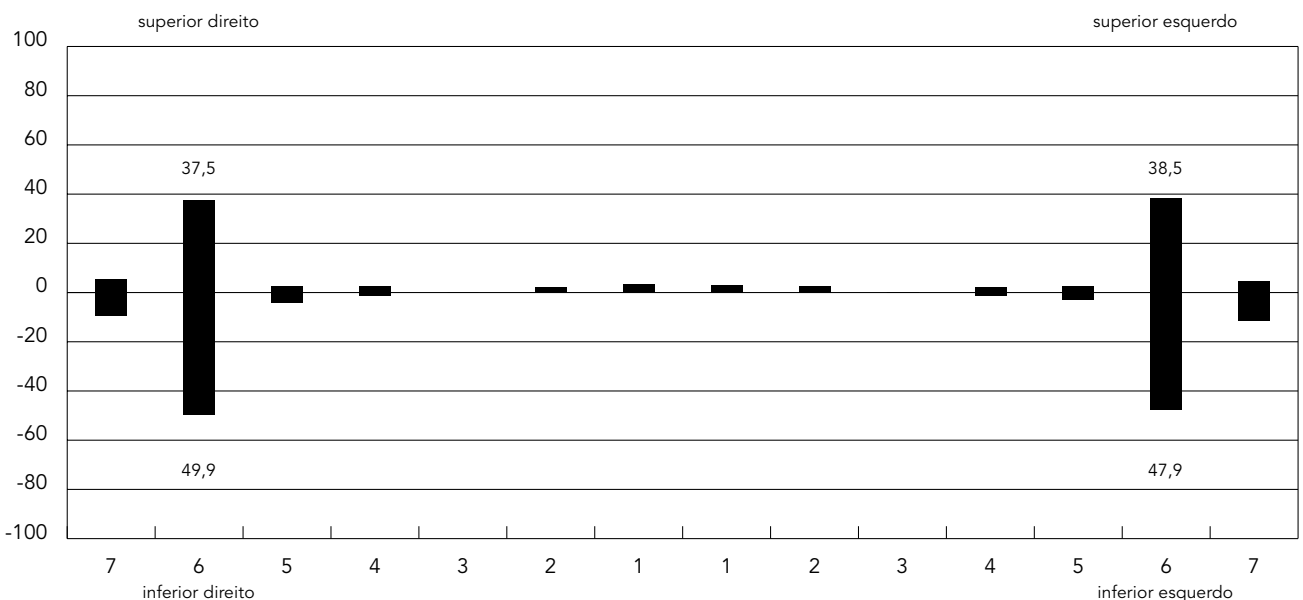

\section{Discussão}

Uma das limitações deste estudo é que não se obteve um número suficiente de elementos amostrais para localidades de prevalência muito baixa, nem tampouco informações de localidades com prevalência muito alta. Entretanto, a amostra final representa diferentes realidades e prevalências de cárie, sendo assim passível de reprodutibilidade. Somandose a isto, este estudo foi realizado por meio de dados secundários e se desconhece quais as dificuldades e/ou facilidades que os examinadores encontrariam se utilizassem a metodologia sugerida pelos índices simplificados durante a coleta dos dados e calibração. Entretanto, tanto Viegas 5 quanto Guimarães 22 encontraram pequenas diferenças entre os valores do índice CPO-D e os valores dos índices simplificados, recomendando a utilização destes para levantamentos epidemiológicos da cárie dentária. 
Figura 2

Proporção de dentes com experiência de cárie dentária, em relação aos dentes irrompidos, em municípios com prevalência moderada de cárie aos 12 anos. Região de Campinas, São Paulo, Brasil, 1998-2001.

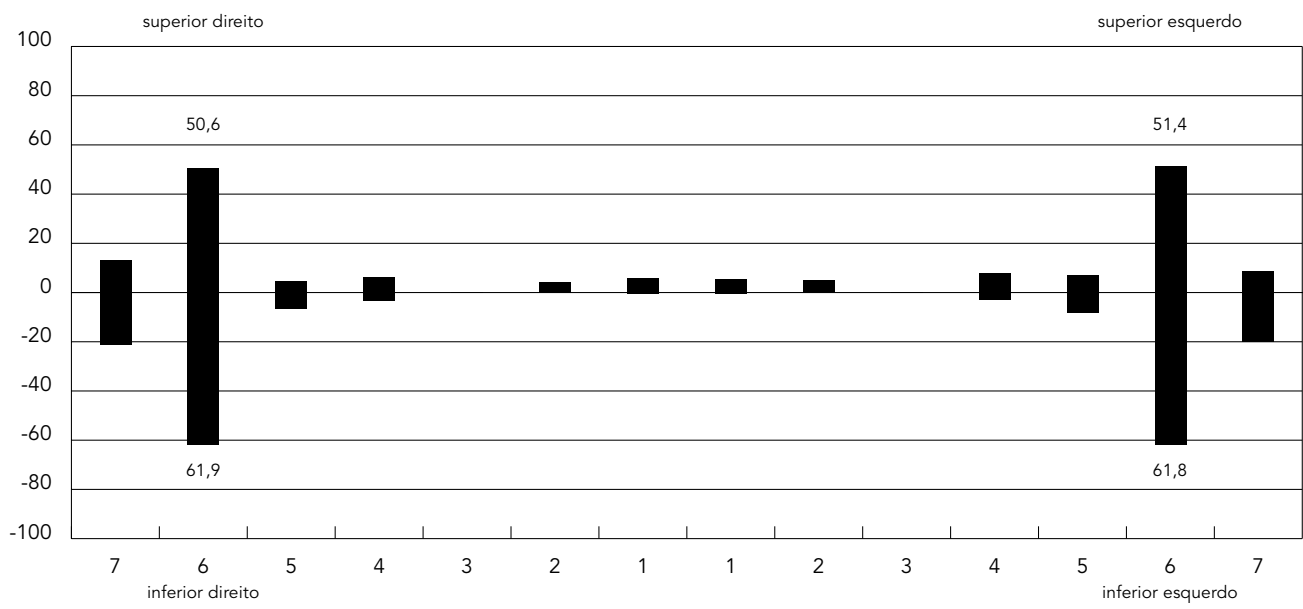

Figura 3

Proporção de dentes com experiência de cárie dentária, em relação aos dentes irrompidos, em municípios com alta prevalência de cárie aos 12 anos. Região de Campinas, São Paulo, Brasil, 1998-2001.

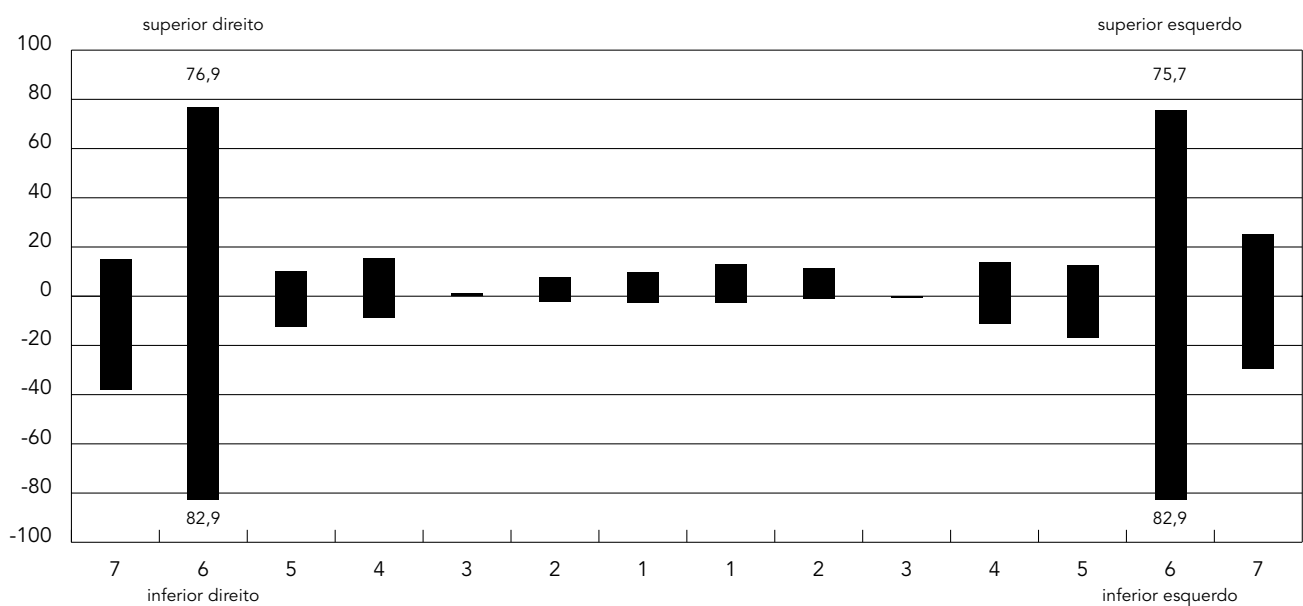


Segundo Pereira 1, os indicadores de saúde devem ser utilizados com o propósito de se conhecer adequadamente a situação de saúde e, por possibilitar comparações individuais ou populacionais, eles podem subsidiar a tomada de decisões racionais e bem fundamentadas. Um outro aspecto interessante que este autor ressalta é que os indicadores apresentam um caráter prognóstico, pois permitem presumir o que poderá ocorrer no futuro, além de constatar as mudanças que ocorrem ao longo do tempo.

Para que isto seja possível, alguns critérios são fundamentais para a seleção e avaliação de indicadores de saúde como validade, reprodutibilidade, representatividade, obediência a preceitos éticos e oportunidade, simplicidade, facilidade de obtenção e custo compatível. Segundo Guimarães 22 e Rodrigues et al. 23, a utilização de índices simplificados também traz como vantagem uma diminuição na discordância de diagnóstico entre os examinadores. Dentro destes parâmetros, os índices simplificados apresentam algumas vantagens quando comparados ao índice CPO-D, e pelos resultados obtidos no presente estudo, a sua aplicação é ainda possível, desde que esteja coerente com os objetivos do estudo.

Entretanto, o índice simplificado MID2 deveria ser aplicado com maior cautela pois apesar da correlação ter sido moderada ou forte, o teste de Wilcoxon mostrou que os valores obtidos em moderada e alta prevalências de cárie são estatisticamente diferentes. Este índice, para a sua obtenção, considera a experiência de cárie dos incisivos superiores, além dos primeiros molares.

Por outro lado, o índice simplificado MID1 mostrou resultados interessantes, pois ao se comparar as médias obtidas por meio do índice simplificado MID1 com o CPO-D nas diferentes prevalências, mostraram pequenas diferenças numéricas, uma correlação moderada e não se verificou diferenças estatisticamente significantes entre os valores, indicando que esse índice simplificado pode ser utilizado para baixa, moderada e alta prevalências de cárie. Destaca-se a simplicidade na sua aplicação, reduzindo consideravelmente o tempo destinado aos exames epidemiológicos. Uma outra vantagem se refere à possibilidade na redução de custos, principalmente quando não há necessidade de um detalhamento da situação de saúde.

A possibilidade da utilização do índice simplificado MID1 pode ser apoiada na evidência de que o primeiro molar inferior continua sendo um bom indicador do nível de cárie de populações jovens devido à hierarquia de ataque da cárie. Sheiham 7 e Poulsen \& Horowitz 24 afirmam que existe uma hierarquia de suscetibilidade da cárie para cada tipo de dente e superfície dental, sendo também apontado por alguns estudos que com a queda da prevalência da cárie dentária, as cáries em sulcos e fissuras começariam a aumentar proporcionalmente e as cáries proximais começariam a ser menos comuns 6,7,25,26. O estudo de Bjarsason et al. 27 confirma esta tendência, pois $64,0 \%$ das lesões de cárie estavam localizadas nas superfícies oclusais das crianças de 12 anos, residentes na Islândia.

Os achados de Silva \& Maltz 28 também são coerentes com os resultados observados no presente estudo, ou seja, há uma forte tendência da cárie se concentrar nos molares e uma reduzida experiência de cárie nos demais grupos dentais.

Por outro lado, Vasconcelos et al. 8, ao verificarem a distribuição da cárie em prevalência muito alta (CPO-D de 8,8), observaram que os incisivos superiores também mostravam uma importante experiência de cárie, assim como os pré-molares. No presente estudo, isto não foi observado, pois a cárie se concentrou nos molares, com valores inferiores a $17,0 \%$ nos demais grupos de dentes (pré-molares, caninos e incisivos). Muito provavelmente observaríamos um padrão semelhante ao descrito por Vasconcelos et al. 8, caso os valores do CPO-D fossem tão altos quanto ao descrito por estes autores.

Os resultados do presente estudo também demonstram que depois dos primeiros molares, a experiência de cárie dos segundos molares foi mais elevada do que a dos incisivos superiores, tanto em alta, moderada ou baixa prevalência de cárie, demonstrando que possivelmente os segundos molares tenham um papel mais importante do que os incisivos em relação à hierarquia de ataque de cárie, resultados estes também apoiados por Sheiham 7, Abernathy et al. 29 e Silva \& Maltz 28.

Desta forma, os resultados do presente estudo permitem concluir que nesta faixa etária da população há uma maior predileção da cárie se localizar nos primeiros molares, que foram os dentes mais afetados, indicando que esses dentes possam ser considerados bons indicadores da experiência de cárie de jovens populações.

Assim, considera-se fundamental o direcionamento de futuros estudos para verificar com maior detalhamento o padrão de distribuição da cárie dentária, considerando diferentes prevalências de cárie e demais variáveis.

Conclui-se que o índice simplificado MID1 pode ser indicado para estudos epidemiológi- 
cos em baixa, moderada e alta prevalências de cárie. Assim, considerando os resultados deste estudo, examinando-se somente o primeiro molar inferior direito é possível estimar quantos dentes por criança, em média, foram atacados pela cárie em uma população. $\mathrm{O}$ índice simplificado MID2 se mostrou mais indicado para localidades com baixa prevalência de cárie, não sendo recomendado para localidades com prevalências moderada e alta de cárie. Ressalta-se ainda que estes índices são mais recomendados para situações onde se objetive um diagnóstico rápido da situação de saúde bucal ou para monitoramentos periódicos.

\section{Resumo}

Este estudo teve como objetivo verificar se dois índices simplificados: "Método Um" e "Método Dois" de Viegas podem ser indicados para levantamentos epidemiológicos em saúde bucal. A amostra foi proveniente de 29 municípios, totalizando 2.378 exames em escolares de 12 anos. Considerando a média CPO-D em cada localidade, obteve-se três grupos de prevalências (baixa, moderada e alta) e nesses grupos foram testados os indices simplificados, calculando-se assim os valores estimados dos índices simplificados. O "Método Um", que se baseia na experiência de cárie do primeiro molar inferior, apresentou correlação moderada nos três grupos de prevalências e semelhança entre as médias $(p<0,05)$. O "Método Dois", que além da experiência de cárie do primeiro molar acrescenta a experiência de cárie dos incisivos centrais superiores, apresentou semelhança com os valores do CPO-D apenas em baixa prevalência de cárie. Conclui-se que o índice simplificado "Método Um" pode ser utilizado em levantamentos epidemiológicos em baixa, moderada e alta prevalências de cárie, evidenciando que o primeiro molar continua sendo um bom indicador do nível de cárie de jovens populações.

Saúde Bucal; Cárie Dentária; Índice CPO

\section{Colaboradores}

Todos os autores contribuíram no processo de elaboração deste artigo científico. S. Cypriano redigiu o corpo do artigo: revisão bibliográfica, introdução, metodologia, resultados e discussão. M. L. R. Sousa e R. S. Wada orientaram e conduziram todas as etapas da pesquisa, colaborando no planejamento e desenho do estudo, discussão dos resultados, análise estatística, conclusão e revisão final do artigo. 


\section{Referências}

1. Pereira MG. Epidemiologia: teoria e prática. Rio de Janeiro: Editora Guanabara Koogan; 2001.

2. Pinto VG. Saúde bucal coletiva. São Paulo: Editora Santos; 2000.

3. Klein H, Palmer CE. Dental caries in the American Indian children. Washington DC: Government Printing; 1938.

4. World Health Organization. Oral health surveys: basic methods. Geneva: World Health Organization; 1997.

5. Viegas AR. Simplified indices for estimating the prevalence of dental caries-experience in children seven to twelve years of age. J Public Health Dent 1969; 29:76-91.

6. McDonald SP, Sheiham A. The distribution of caries on different tooth surfaces at varying levels of caries - a compilation of data from 18 previous studies. Community Dent Oral Epidemiol 1992; 9:39-48.

7. Sheiham A. Impact of dental treatment on the incidence of dental caries in children and adults. Community Dent Oral Epidemiol 1997; 25:104-12.

8. Vasconcellos MCC, Jesus BJ, Nogueira JRB, Lui Filho O. Distribuição de cárie dentária na dentição permanente de escolares: experiência por dente. Rev Odontol Univ São Paulo 1994; 8:125-30.

9. Bastos JLD, Nomura LH, Peres MA. Tendência de cárie dentária em escolares de 12 e 13 anos de idade de uma mesma escola no período de 1971 a 2002, em Florianópolis, Santa Catarina, Brasil. Cad Saúde Pública 2004; 20:117-22.

10. Bönecker M, Cleaton-Jones P. Trends in dental caries in Latin American and Caribbean 5-6 and 11-13-year-old children: a systematic review. Community Dent Oral Epidemiol 2003; 31:152-7.

11. Pitts NB, Evans DJ, Nugent ZJ, Pine CM. The dental caries experience of 12-year-old children in England and Wales. Surveys coordinated by the British Association for the Study of Community Dentistry in 2000/2001. Community Dent Health 2002; 19:46-53.

12. Narvai PC, Castellanos RA, Frazão P. Prevalência de cárie em dentes permanentes de escolares no município de São Paulo, SP, 1970-1996. Rev Saúde Pública 2000; 34:196-200.

13. Dini EL, Foschini ALR, Brandão IMG, Silva SRC Changes in caries prevalence in 7-12 year-old children from Araraquara, São Paulo, Brazil: 19891995. Cad Saúde Publica 1999; 15:617-21.

14. Freire MCM, Pereira MF, Batista SMO, Borges MRS, Barbosa MIB, Rosa AGF. Prevalência de cárie e necessidade de tratamento em escolares de 6 a 12 anos da rede pública de ensino. Rev Saúde Pública 1999; 33:385-90.
15. Peres MAA, Narvai PC, Calvo MC. Prevalência de cárie dentária em crianças aos doze anos de idade, em localidades do Estado de São Paulo, Brasil, período 1990-1995. Rev Saúde Pública 1997; 31: 594-600.

16. Petersson HG, Bratthall D. The caries decline: a review of reviews. Eur J Oral Sci 1996; 104:436-43.

17. Marthaler TM, O'Mullane DM, Vrbic V. The prevalence of dental caries in Europe 1990-1995. Caries Res 1996; 30:237-55.

18. Murray JJ. O uso correto de fluoretos na saúde pública. São Paulo: Editora Santos; 1992.

19. Silva NN. Amostragem probabilística: um curso introdutório. São Paulo: Edusp; 2001.

20. Siegel S. Estatística não paramétrica (para as ciências do comportamento). São Paulo: McGrawHill do Brasil; 1981.

21. Rowntree D. Statistics without tears: a primer for non-mathematicians. London: Penguin Books; 1981.

22. Guimarães LOC. Contribuição para o estudo da bilateralidade da cárie dentária em dentes permanentes. Rev Fac Odontol São Paulo 1971; 9:3118.

23. Rodrigues CRMD, Ando T, Guimarães LOC. Simplificação do índice de cárie nas idades de 4 a 6 e de 7 a 10 anos (dentições decídua e mista). Rev Odontol Univ São Paulo 1989; 3:454-9.

24. Poulsen S, Horowitz HS. An evaluation of hierarchical method of describing the pattern of dental caries attack. Community Dent Oral Epidemiol 1974; 2:7-11.

25. Burt BA. Prevention policies in the light of the changed distribution os dental caries. Acta Odontol Scand 1998; 56:179-86.

26. Jackson D, James PMC, Wolfe WB. Fluoridation in Alglesey. Br Dent J 1975; 138:165-71.

27. Bjarnason S, Finnbogason SY, Holbrook P, Köhler B. Caries experience in Icelandic 12-year-old urban children between 1984 and 1991. Community Dent Oral Epidemiol 1993; 21:194-7.

28. Silva BB, Maltz M. Prevalência de cárie, gengivite e fluorose em escolares de 12 anos de Porto Alegre - RS, Brasil, 1998/1999. Pesqui Odontol Bras 2001; 15:208-14.

29. Abernathy JR, Graves RC, Greenberg BG, Bohannan HM, Disney JA. Application of life table methodology in determining dental caries rates. Community Dent Oral Epidemiol 1986; 14: 261-4.

Recebido em 17/Nov/2003

Versão final reapresentada em 07/Abr/2004 Aprovado em 29/Jun/2004 